flo/abu/marek

\title{
Concurrence of mixed bipartite quantum states in arbitrary dimensions
}

\author{
Florian Mintert ${ }^{1,2}$, Marek Kuś ${ }^{2}$, and Andreas Buchleitner ${ }^{1}$ \\ ${ }^{1}$ Max-Planck-Institut für Physik komplexer Systeme, Nöthnitzerstr. 38, D-01187 Dresden and \\ ${ }^{2}$ Centrum Fizyki Teoretycznej, Polska Akademia Nauk, Al. Lotników 32/44, PL-02-668 Warszawa
}

(Dated: September 3, 2021)

\begin{abstract}
We derive a lower bound for the concurrence of mixed bipartite quantum states, valid in arbitrary dimensions. As a corollary, a weaker, purely algebraic estimate is found, which detects mixed entangled states with positive partial transpose.

PACS numbers: 03.67.-a, 03.67.Mn, 89.70.+c
\end{abstract}

In classical physics, one can always divide a system into subsystems, such that complete information on the entity implies a complete description of its individual parts, and vice versa. In quantum physics, this no longer holds true: whilst one can still divide a system into subsystems, a complete description of the system state in terms of a pure state does not necessarily assign a pure state to each subsystem. The subsystems of generic pure states are correlated in a way without classical analog - they are entangled.

While such quantum entanglement arguably incarnates the key difference between the quantum and the classical world, and is nowadays understood as a resource in various tasks of quantum information processing [1] such as cryptography, teleportation, and quantum computation, it remains hard to quantify, for arbitrary quantum states [2]. In particular, when coupled to an environment, pure quantum states rapidly evolve into mixed states which bear entanglement together with classical probabilistic correlations, and the latter have to be distinguished from the former. Furthermore, the complete characterization of the nonclassical correlations of a given state becomes an ever more complex task as the Hilbert space dimension increases, thus turning into a computationally extremely intricate problem.

No equally versatile as computationally manageable entanglement measure for mixed states is available so far, although various more or less pragmatically motivated quantities have been proposed. The most popular indicator of entanglement is the positive partial transpose (ppt) criterion [3] and variants thereof, such as negativity [4], though these do not reliably detect arbitrary entangled states. Another approach for quantifying entanglement is through entanglement witnesses [5] which, however, need to be constructed anew for each given quantum state, and such construction can be rather involved [6]. Finally, there are mixed state generalizations of pure state entanglement measures [7, 8, 9, 10, 11], which, in general, require a high dimensional optimisation procedure. By construction, any numerical evaluation of these latter quantities only yields upper bounds for the entanglement of a given state but cannot reliably distinguish it from separable states, let alone provide a reliable quantitative estimate of the state's actual degree of entanglement.

In the present Letter, we improve on that situation: We derive a lower bound of concurrence [7, 9] - a quantity which is strictly larger than zero for nonvanishing entanglement - of mixed bipartite quantum states in arbitrary dimensions. Our bound is given by a purely algebraic expression which is readily evaluated for arbitrary states, and can be tightened numerically on a relatively lowdimensional parameter space, of reduced dimension as compared to hitherto available optimisation procedures 12]. This complements already available upper bounds 10, 11] and provides, for the first time, a rather precise estimate of the actual value of concurrence.

We start out with the definition [11] of a pure state's concurrence as $c(\psi)=\sqrt{|\langle\psi \mid \psi\rangle|^{2}-\operatorname{Tr} \varrho_{r}^{2}}$, where the reduced density matrix $\varrho_{r}$ is obtained by tracing over one subsystem. The concurrence of mixed states $\varrho$ is then given as the convex roof

$$
c(\varrho)=\inf \sum_{i} p_{i} c\left(\Psi_{i}\right), \varrho=\sum_{i} p_{i}\left|\Psi_{i}\right\rangle\left\langle\Psi_{i}\right|, \quad p_{i} \geq 0,
$$

of all possible decompositions into pure states $\left|\Psi_{i}\right\rangle$. Consequently, $c(\varrho)$ vanishes if and only if $\varrho$ exhibits purely classical correlations, i.e. if the state is separable and hence can be represented as a convex sum over product states, $\varrho=\sum_{i} p_{i} \varrho_{i}^{(1)} \otimes \varrho_{i}^{(2)}$, with $p_{i} \geq 0$, and $\varrho_{i}^{(1)}$ and $\varrho_{i}^{(2)}$ states on the subcomponents $\mathcal{H}_{1}$ and $\mathcal{H}_{2}$ of the total Hilbert space $\mathcal{H}=\mathcal{H}_{1} \otimes \mathcal{H}_{2}$. Given the dimensions $n_{1}$ and $n_{2}$ of $\mathcal{H}_{1}$ and $\mathcal{H}_{2}$, respectively, eq. (1) defines a high dimensional optimisation problem which is rather cumbersome to solve. Furthermore, as already mentioned above, such optimisation can only yield an upper bound for $c(\rho)$, by virtue of the definition of the infimum.

To estimate $c(\rho)$ from below, we first replace, for convenience, the $\left|\Psi_{i}\right\rangle$ by the subnormalized states $\left|\psi_{i}\right\rangle=$ $\sqrt{p_{i}}\left|\Psi_{i}\right\rangle$ in eq. (11). Given a valid decomposition $\left\{\left|\phi_{i}\right\rangle\right\}$ of $\varrho$ into subnormalized states, any other suitable set $\left\{\left|\psi_{i}\right\rangle\right\}$ is obtained [13] by transformations $V \in \mathbf{C}^{N \times r}$, with $r$ and $N$ the lengths of the sets $\left\{\left|\phi_{i}\right\rangle\right\}$ and $\left\{\left|\psi_{i}\right\rangle\right\}$, respectively,

$$
\left|\psi_{i}\right\rangle=\sum_{j=1}^{r} V_{i j}\left|\phi_{j}\right\rangle, \sum_{i=1}^{N} V_{k i}^{\dagger} V_{i j}=\delta_{j, k}
$$

It is now crucial to realize that the concurrence of a pure state $|\psi\rangle$ can be expressed as the square root of the func- 
tion

$$
\begin{gathered}
f\left(\psi_{1}, \psi_{2}, \psi_{3}, \psi_{4}\right)=\left\langle\psi_{2} \mid \psi_{1}\right\rangle\left\langle\psi_{4} \mid \psi_{3}\right\rangle- \\
\operatorname{Tr}_{1}\left(\left(\operatorname{Tr}_{2}\left|\psi_{1}\right\rangle\left\langle\psi_{2}\right|\right)\left(\operatorname{Tr}_{2}\left|\psi_{3}\right\rangle\left\langle\psi_{4}\right|\right)\right),
\end{gathered}
$$

evaluated at $\psi=\psi_{1}=\psi_{2}=\psi_{3}=\psi_{4}$, where $\operatorname{Tr}_{1}$ and $\operatorname{Tr}_{2}$ denote the traces over the first and the second subsystem. $f$ is linear in its first and third, and anti-linear in the second and fourth argument. Due to these properties the definition (11) can be reformulated as an infimum over transformations $V$ :

$$
c(\varrho)=\inf _{V} \mathcal{C}, \quad \text { with } \quad \mathcal{C}=\sum_{i=1}^{N}\left(\left[V \otimes V A V^{\dagger} \otimes V^{\dagger}\right]_{i i}^{i i}\right)^{\frac{1}{2}} .
$$

Herein, the tensor $A$, defined by $A_{j k}^{l m}=f\left(\phi_{j}, \phi_{l}, \phi_{k}, \phi_{m}\right)$ 14] is hermitian, $A_{j k}^{l m}=\left(A_{l m}^{j k}\right)^{*}$, and symmetric with respect to a simultaneous exchange of both its co- and contravariant indices $A_{j k}^{l m}=A_{k j}^{m l}$. Due to the symmetry of the transformation $V \otimes V$ under exchange of the subsystems of $A$, we can replace $A_{j k}^{l m}$ in eq. (4) by the symmetrised elements

$$
\mathcal{A}_{j k}^{l m}=\frac{1}{2}\left(A_{j k}^{l m}+A_{k j}^{l m}\right),
$$

which is equivalent to a symmetrisation over both subsystems in eq. (3). It can be shown that $\mathcal{A}$ is positive semidefinite and that its support lies in an antisymmetric subspace, i.e., all elements of $\mathcal{A}$ with respect to fully symmetric linear combinations of product states vanish. Since the antisymmetric subspace has dimension $m=n_{1}\left(n_{1}-1\right) n_{2}\left(n_{2}-1\right) / 4, \mathcal{A}$ has at most $m$ nonvanishing eigenvalues.

Due to the discussed symmetries $\mathcal{A}$ can be expanded in a basis of real symmetric matrices $\Lambda^{\alpha} \in \mathbf{R}^{r \times r}$

$$
\mathcal{A}_{j k}^{l m}=\sum_{\alpha, \beta} B_{\alpha \beta} \Lambda_{j k}^{\alpha} \Lambda_{l m}^{\beta}
$$

with $B$ hermitian and positive semi definite. With the eigenvalues and associated eigenvectors of $B\left(B \vec{x}^{\alpha}=\right.$ $\mu_{\alpha} \vec{x}^{\alpha}$ and $\left.\vec{x}^{\alpha}=\left[x_{1}^{\alpha}, \ldots, x_{i}^{\alpha}, \ldots\right]\right)$ we can construct a properly normalised eigensystem $T^{\alpha}$ of $\mathcal{A}$

$$
T_{\alpha}=\sqrt{\mu_{\alpha}} \sum_{\beta} x_{\beta}^{\alpha} e^{i \phi_{\alpha}} \Lambda^{\beta}=\mathcal{T}_{\alpha} e^{i \phi_{\alpha}}, \alpha=1, \ldots, m .
$$

We explicitly take into account the free phase factors $\exp \left(i \phi_{\alpha}\right)$, as they will be crucial in the following. Consequently,

$$
\mathcal{A}_{j k}^{l m}=\sum_{\alpha} T_{j k}^{\alpha}\left(T_{l m}^{\alpha}\right)^{*}=\sum_{\alpha} \mathcal{T}_{j k}^{\alpha}\left(\mathcal{T}_{l m}^{\alpha}\right)^{*} .
$$

Hence, eq. (4) can now be rewritten as

$$
\mathcal{C}=\sum_{i=1}^{N}\left(\sum_{\alpha}\left|\left[V T^{\alpha} V^{T}\right]_{i i}\right|^{2}\right)^{\frac{1}{2}}
$$

the infimum of which gives the concurrence of the mixed state $\varrho$.

Note that eq. (9) resembles the concurrence vector introduced in [15], a quantity with elements analogous to $\sum_{i=1}^{N}\left|\left[V T^{\alpha} V^{T}\right]_{i i}\right|$. In [15] it was shown that the concurrence vector vanishes identically, for suitably chosen $V$, if and only if $\varrho$ is separable, thus providing a separability criterion. Whilst the very same equivalence holds for vanishing $\mathcal{C}$, the explicit expression (9) allows us to proceed further: The Cauchy-Schwarz inequality $\left(\sum_{\alpha} x_{\alpha}^{2}\right)^{\frac{1}{2}}\left(\sum_{\alpha} y_{\alpha}^{2}\right)^{\frac{1}{2}} \geq \sum_{\alpha} x_{\alpha} y_{\alpha}$, and $\sum_{\alpha}\left|z_{\alpha}\right| \geq$ $\left|\sum_{\alpha} z_{\alpha}\right|$, for $z_{\alpha} \in \mathbf{C}$, imply

$$
c(\varrho) \geq \inf _{V} \sum_{i=1}^{N}\left|\left[V\left(\sum_{\alpha} z_{\alpha} \mathcal{T}^{\alpha}\right) V^{T}\right]_{i i}\right|,
$$

for any set $z_{\alpha}=y_{\alpha} \exp \left(i \phi_{\alpha}\right)$, with $y_{\alpha} \geq 0, \sum_{\alpha} y_{\alpha}^{2}=1$. The infimum on the rhs is given by $\lambda_{1}-\sum_{i>1} \lambda_{i}$, where $\lambda_{j}$ are the singular values of $\mathcal{T}=\sum_{\alpha} z_{\alpha} \mathcal{T}_{\alpha}$, i.e., the square roots of the eigenvalues of the positive hermitian matrix $\mathcal{T T}^{\dagger}$ in decreasing order [10]. Hence, we arrive at the desired lower bound,

$$
c(\varrho) \geq \lambda_{1}-\sum_{i>1} \lambda_{i}
$$

with the $\lambda_{j}$ dependent on the choice of the $y_{\alpha}$ and $\phi_{\alpha}$.

Note that each set $\left\{y_{\alpha}, \phi_{\alpha}\right\}$ provides a lower bound of $c(\varrho)$, which can be tightened by numerical optimization. However, all the examples we have considered so far suggest that there is one matrix $\mathcal{T}^{\alpha}$ that gives the main contribution to the rhs of eq. (10). Hence, the singular values of this matrix provide a purely algebraic lower bound for $c$, which often leads to satisfactory results even without further numerical refinement. As an example, consider the family of two spin-1 states

$$
\varrho_{a}=\frac{1}{1+8 a}\left[\begin{array}{ccccccccc}
a & 0 & 0 & 0 & a & 0 & 0 & 0 & a \\
0 & a & 0 & 0 & 0 & 0 & 0 & 0 & 0 \\
0 & 0 & a & 0 & 0 & 0 & 0 & 0 & 0 \\
0 & 0 & 0 & a & 0 & 0 & 0 & 0 & 0 \\
a & 0 & 0 & 0 & a & 0 & 0 & 0 & a \\
0 & 0 & 0 & 0 & 0 & a & 0 & 0 & 0 \\
0 & 0 & 0 & 0 & 0 & 0 & \beta & 0 & \gamma \\
0 & 0 & 0 & 0 & 0 & 0 & 0 & a & 0 \\
a & 0 & 0 & 0 & a & 0 & \gamma & 0 & \beta
\end{array}\right], a \in[0,1]
$$

with $\beta=(1+a) / 2$ and $\gamma=\sqrt{1-a^{2}} / 2$, described in [16]: The state $\varrho_{a}$ has positive partial transpose in the entire range of $a$, i.e., the standard criterium [3] identifying a mixed state as nonseparable via negativity of its partial transpose is inoperational here. Notwithstanding, the singular values of $\mathcal{T}^{1}$ associated with the largest eigenvalue of $B$ in eq. (6) above already provide a positive lower bound $c_{l}^{(1)}=\lambda_{1}^{(1)}-\sum_{i>1} \lambda_{i}^{(1)}$ for $c\left(\varrho_{a}\right)$, as shown in Fig. 1 $\varrho_{a}$ is detected as entangled without any need for further numerical optimisation. However, the figure also shows that taking into account all $\mathcal{T}^{\alpha}$, numerical optimisation significantly raises the lower bound. The 


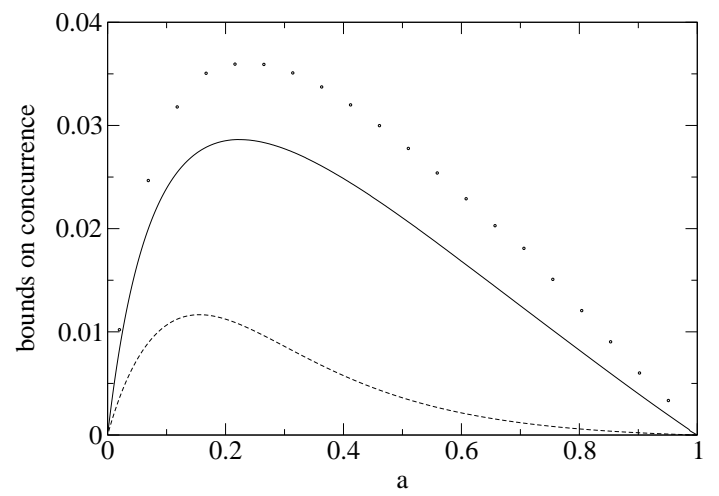

FIG. 1: Lower bound (full line) of the concurrence $c$ of the family of two spin-1 states $\varrho_{a}$ [16], together with its purely algebraic approximation $c_{l}^{(1)}=\lambda_{1}^{(1)}-\sum_{i>1} \lambda_{i}^{(1)}$ (dashed line), over the entire parameter range $a=0 \ldots 1$. Whilst $\varrho_{a}$ has positive partial transpose and is therefore not detected via the negative partial transpose criterion for entanglement, already the algebraic approximation of our lower bound indentifies the state as nonseparable, without need for numerical optimisation, for all $a$. Optimal lower bound and optimal upper bound (dotted line, obtained by minimizing $\mathcal{C}$ in (4) [1]]) confine the actual value of concurrence to an interval with relative uncertainty of the order of approx. $10 \%$.

remaining gap with respect to the upper bound obtained from numerical minimization of $\mathcal{C}$ in (4) leaves a relative uncertainty of the order of approx. $10 \%$ on the actual value of $c(\varrho)$, in the particularly pathological test case considered here.

Let us finally note that the original definition of concurrence [9] is nicely embedded in our formalism. In the case of two-level systems one has $m=1$, i.e., there is only one non-vanishing matrix $T^{1}$. Therefore, eq. (9) simplifies to

$$
\mathcal{C}=\sum_{i=1}^{N}\left|\left[V T^{1} V^{T}\right]_{i i}\right|,
$$

and the infimum can be derived analytically. Indeed, it is found that $T^{1}$ coincides with $\tau$ defined in the original work 9 ].

In conclusion, we have shown that a suitable representation of the concurrence of bipartite mixed quantum states in terms of the eigensystem of a tensorial quantity allows for the derivation of a lower bound of $c(\varrho)$, for arbitrary $\rho$. Not only can this bound be tightened by an optimisation under the comparatively simple constraint $\sum_{\alpha}\left|z_{\alpha}\right|^{2}=1$, over a complex vector space of dimension $n_{1}\left(n_{1}-1\right) n_{2}\left(n_{2}-1\right) / 4$ - at least a factor $4 n_{1} n_{2}$ smaller than dimensions of optimisation procedures hitherto available. It also can be reduced to a purely algebraic bound which appears to provide good estimates, according to numerical data which complement our analytical work. Last but not least, our result can serve to derive lower bounds on the entanglement of formation $E[\underline{8}]$ of a mixed quantum state, thus quantifying the minimally required resources to prepare $\varrho$ : Given any monotonously increasing, convex function $\mathcal{E}$ which satisfies $\mathcal{E}(c(\psi)) \leq-\operatorname{Tr} \varrho_{r} \log \varrho_{r}$, it follows that $E(\varrho) \geq \mathcal{E}(c(\varrho))$, with the rhs bounded from below by our bound (11).

We are indebted to André Ricardo Ribeiro de Carvalho, Rafał Demkowicz-Dobrzański and Karol Życzkowski for fruitful discussions, comments and remarks. Financial support by VolkswagenStiftung and the Polish Ministery of Science through the grant No. PBZ-MIN-008/P03/2003 is gratefully acknowledged.
[1] M. A. Nielsen and I. L. Chuang, Quantum computation and quantum information (Cambridge University Press, 2000).

[2] D. Bruß, J. Math. Phys. 43, 4237 (2002).

[3] A. Peres, Phys. Rev. Lett. 77, 1413 (1996).

[4] G. Vidal and R. F. Werner, Phys. Rev. A 65, 032314 (2002).

[5] B. Terhal, Phys. Lett. A 271, 319 (2000).

[6] A. C. Doherty, P. A. Parrilo, and F. M. Spedalieri, Phys. Rev. Lett. 88, 187904 (2002).

[7] L. P. Hughston, R. Josza, and W. K. Wootters, Phys. Lett. A 183, 14 (1993).

[8] C. H. Bennet, D. P. DiVincenzo, J. Smolin, and W. K. Wootters, Phys. Rev. A 54, 3824 (1996).

[9] W. K. Wootters, Phys. Rev. Lett. 80, 2245 (1998).
[10] A. Uhlmann, Phys. Rev. A 62, 032307 (2000).

[11] P. Rungta, V. Buzek, C. M. Caves, M. Hillery, G. J. Milburn, and W. K. Wootters, Phys. Rev. A 64, 042315 (2001).

[12] A. Uhlmann, Open Sys. Info. Dyn. 5(3), 209 (1998).

[13] E. Schrödinger, Proc. Cambridge Philos. Soc 32, 446 (1936).

[14] P. Badzia̧g, P. Deuar, M. Horodecki, P. Horodecki, and R. Horodecki, J. Mod. Opt 49, 1289 (2002).

[15] K. Audenaert, F. Verstraete, and B. D. Moor, Phys. Rev. A 64, 052304 (2001).

[16] P. Horodecki, Phys. Lett. A 232, 333 (1997).

[17] F. Mintert, Ph.D. thesis, Ludwig-MaximiliansUniversität München (submitted) (2004). 